\title{
STUDY OF MINIMAL EFFECTIVE REEFING RATIO BASED ON AN EMPIRICAL FORMULA AND FLUID-STRUCTURE-INTERACTION METHOD
}

\author{
Xiaoshun ZHAO, Xue YANG, Li YU*, Xin LIU, Jingjing WANG \\ College of Aerospace Engineering, Nanjing University of Aeronautics and Astronautics, Nanjing, China
}

Received 21 June 2018; accepted 23 September 2018

\begin{abstract}
The reefing ratio for the first stage of a parachute limits the reefing ratio for the subsequent stages, so its minimal effective value is very important. In this paper, an empirical formula is derived to calculate the minimal effective reefing ratio. The empirical parameters are obtained by the arbitrary Lagrangian-Eulerian/fluid-structure interaction (ALE/FSI) method. By using the FSI method, the typical flow and structure fields of effective and ineffective reefed parachutes are revealed. The numerical results including drag characteristics and final shape are very consistent with wind tunnel tests. The curves of the empirical parameters with reefing ratios are obtained. The minimal effective reefing ratio obtained by the empirical formula is consistent with that of the numerical results, which shows that the empirical formula has high accuracy.
\end{abstract}

Keywords: reefed parachute system, fluid-structure interaction, reefing ratio, empirical formula, numerical simulation.

\section{Introduction}

The reefing technique is used in the inflation process of parachutes that carry a payload and are operated at high speed to control the drag characteristics. The reefing ratio, which is the length of the reefing line divided by the length of the parachute skirt, is used to represent the reefing degree (Wang, 1997). The study of reefed parachutes began in the late 1940s (Manley \& Butler, 1999). With the development of the work scope of the parachute, multi-stage reefed parachutes became widely used to reduce opening load and loiter time. In the late 1970s, the reefed parachute was used in many deceleration systems. The two-stage reefing technique was applied in the Apollo (Holt, 1968; Robert, 1973; Runkle \& Wolf, 1995). The five-stage reefing technique was applied in the X-38 CRV deceleration parachute (Behr, Wolf, \& Rutledge, 2001). In the Crew Exploration Vehicle Parachute Assembly System (CAPS) parachute system, the extraction parachute has two reefed stages, and the main parachute has three stages (Bledsoe, Englert, Morris, \& Olmstead, 2009; Jose \& Eric, 2013). The greater the number of reefed stages, the smaller the value range of the first stage reefing ratio. If the reefing ratio is too small, the air inflow is not enough to make the canopy form a stable aerodynamic profile. Then the reefed parachute cannot provide an effective drag force. The reefed time is always within the tens of seconds. The safety of the whole recovery system is threatened by an ineffective reef, which is always avoided by increasing the reefing ratio. However, for the multi-stage reefed parachute, if the reefing ratio of the first stage is too high, the reefing ratios of the other stages will each have a narrow optional range. So, the design opening load cannot be achieved. Formulating a technique to find the minimal effective reefing ratio is an important problem that is yet to be resolved.

In recent years, some studies have been carried out on the FSI numerical simulation of the reefed parachute. In 2009, Jason (2009) simulated the flow field of a ringsail parachute with four effective reefing ratios by the deformingspatial-domain/stabilized space-time (DSD/SST) coupling method. Then Kenji, Tayfun, and Cody (2014) further extended this method to simulate the disreefing process. A new contact algorithm and homogenized assumption were introduced to take the dramatically changing shape into consideration. Afterwards, the computational fluid dynamics/mass spring damper (CFD/MSD) model was developed by Tang and Qian (2015) to simulate the inflation process of a reefed parachute. In this model, the parachute is dispersed into a series of mass points connected by springs and dampers. The movement of each mass point is controlled

*Corresponding author. E-mail: yuli_happy@163.com

This is an Open Access article distributed under the terms of the Creative Commons Attribution License (http://creativecommons.org/licenses/by/4.0/), which permits unrestricted use, distribution, and reproduction in any medium, provided the original author and source are credited. 
by the flow field force, spring force, and damping force. The dynamic changing process of the two-dimensional flow field is analyzed in this paper. The asymmetry of the flow and structure field is eliminated using some simplifying assumptions described in the methods above. The influence of the reefing ratio on the inflation of the ringsail parachute has been researched by the arbitrary Lagrangian-Eulerian/ fluid-structure interaction (ALE/FSI) (Gao \& Yu, 2014; Cheng, Yu, Rong, \& Jia, 2012a). Numerical results including canopy shape, opening load, drag characteristics, and swing angle, were very consistent with wind tunnel tests. The relationships between the reefing ratio and the maximum projected diameter, drag area, and maximum opening load, were obtained.

In summary, most previous works on reefed parachutes describe simulations or experiments on parachute systems with specified reef ratios. An ineffective reefing ratio has not been considered. A major aim of this paper is to discuss approaches and strategies to find the smallest effective reefing ratio. This paper is organized as follows. In the next section, the theory of ALE/FSI and an empirical formula to calculate the smallest effective reefing ratio are described. The geometry and FSI models of reefed parachutes are presented in Section 3. In Section 4, the numerical results are compared with the experimental data measured by the China Academy of Space Technology (CAST). Additionally, numerical examples with 7 reefing ratios, 4 dynamic pressures, and 2 vent diameters are simulated by the FSI method to discuss the performance of the empirical formula. Finally, conclusions and future work are summarized in Section 5.

\section{Governing equations}

\subsection{Governing equations of flow and structure field}

The governing equations of the flow field for the ALE description are as follows.

Mass equation:

$$
\frac{\partial \rho}{\partial t}=-\rho \frac{\partial v_{i}}{\partial x_{i}}-w_{i} \frac{\partial \rho}{\partial x_{i}} .
$$

Momentum equation:

$$
\rho \frac{\partial v_{i}}{\partial t}=\sigma_{i j, j}+\rho b_{i}-\rho w_{j} \frac{\partial v_{i}}{\partial x_{j}} .
$$

Energy equation:

$$
\rho \frac{\partial E}{\partial t}=\sigma_{i j} v_{i . j}+\rho b_{i} v_{i}-\rho w_{j} \frac{\partial E}{\partial x_{j}} .
$$

In equations (1)-(3), $v_{i}$ and $w_{i}$ are the material and reference velocities, respectively, $b_{i}$ describes the body force, and $\sigma_{i j}$ is the stress tensor, here:

$$
\sigma_{i j}=-p \delta_{i j}+\mu\left(v_{i . j}+v_{j . i}\right)
$$

and $\delta_{i j}$ is the Kronecker's delta function. The relationship of the Lagrangian, Eulerian, and referential coordinates is

$$
\frac{\partial f\left(X_{i}, t\right)}{\partial t}=\frac{\partial f\left(x_{i}, t\right)}{\partial t}+w_{i} \frac{\partial f\left(x_{i}, t\right)}{\partial t},
$$

where $X_{i}$ is the Lagrangian coordinate, and $x_{i}$ is the Eulerian coordinate.

The governing equations of the structure field for the ALE description are as follows.

$$
\rho_{s} \frac{d^{2} u_{i}}{d t^{2}}=\sigma_{i j . j}+\rho_{s} b_{i},
$$

where $u_{i}$ is the displacement, and $\rho_{s}$ is the density of the structure.

The Second Piola-Kirchoff stress tensor $S_{i j}$ and the Green strain tensor $E_{k l}$ have been employed to describe the geometrical nonlinear system based on the fourthorder elastic tensor $C_{i j k l}$ :

$$
S_{i j}=C_{i j k l} E_{k l} \text {. }
$$

In this paper, the fabric is simulated by the three-node shell element. It only contains in-plane stress. The material is isotropic, so $S_{i j}$ can be simplified as:

$$
\left\{\begin{array}{l}
S_{11} \\
S_{22} \\
S_{12}
\end{array}\right\}=\frac{E}{(1+v)(1-2 v)}\left[\begin{array}{ccc}
1-v & v & 0 \\
v & 1-v & 0 \\
0 & 0 & \frac{1-2 v}{2}
\end{array}\right]\left\{\begin{array}{l}
E_{11} \\
E_{22} \\
E_{12}
\end{array}\right\},
$$

where $v$ is the Poisson ratio and $E$ is the Young's modulus.

The numerical discrete of the fluid and structure field can be written as:

$$
\begin{aligned}
& \boldsymbol{M} \cdot{ }_{k+1}^{t+\Delta t} \ddot{U}+\boldsymbol{C} \cdot{ }_{k+1}^{t+\Delta t} \dot{U}+{ }_{k}^{t+\Delta t} \boldsymbol{K}_{\boldsymbol{T}}\left[{ }_{k+1}^{t+\Delta t} U-{ }_{k}^{t+\Delta t} U\right]= \\
& { }_{k+1}^{t+\Delta t} \boldsymbol{F}-{ }_{k}^{t+\Delta t} \boldsymbol{R}
\end{aligned}
$$

where $\boldsymbol{M}$ is the consistent mass matrix, $\boldsymbol{C}$ is the mass damping matrix, $K_{T}$ is the tangent stiffness matrix, and $U$ is the global displacements vector. $\boldsymbol{F}$ and $\boldsymbol{R}$ are the pressure loading and restoring force, respectively. Superscript $t$ is the physical time step and subscript $k$ is the NewtonRaphson sub iteration step number.

\subsection{Coupling method}

The velocity and displacement of the structure and flow field in the coupling domain are updated by

$$
\begin{aligned}
& \boldsymbol{u}^{n+1 / 2}=\boldsymbol{u}^{n-1 / 2}+\Delta t \cdot \boldsymbol{M}^{-1} \cdot\left(\boldsymbol{F}_{\text {ext }}+\boldsymbol{F}_{\text {int }}\right), \\
& \boldsymbol{x}^{n+1}=\boldsymbol{x}^{n}+\Delta t \cdot \boldsymbol{u}^{n+1 / 2},
\end{aligned}
$$

where $\boldsymbol{F}_{\text {ext }}$ and $\boldsymbol{F}_{\text {int }}$ are the internal and external forces, and $\boldsymbol{M}$ is the mass matrix. The structure and flow field are coupled by the penalty function. The interface force is calculated based on

$$
\boldsymbol{F}_{c}=k \boldsymbol{d},
$$

where $\boldsymbol{d}$ is the distance of the coupling point, and $k$ is the stiffness coefficient. In order to achieve coupling, the 
speed and displacement in the coupling domain are adjusted based on $\boldsymbol{F}_{c}$, which is a part of $\boldsymbol{F}_{\text {ext }}$.

\subsection{Empirical formula for the minimal effective reefing ratio}

As shown in Figure 1, the aerodynamic shape of a reefed parachute with an effective reefing ratio is approximately composed of a hemisphere at the top and a frustum at the bottom. When the air is incompressible, the change of the canopy volume can be written as

$$
\begin{aligned}
& \frac{\mathrm{d} V}{\mathrm{~d} t}=n_{1} \pi r_{1}^{2} v_{\infty}-\left(2 \pi r_{2}^{2}-\pi r_{d}^{2}\right) v_{q}-n_{2} \pi r_{d}^{2} v_{\infty}- \\
& n_{3} v_{\infty} 2 \pi r_{2}^{2} \alpha
\end{aligned}
$$

where $v_{\infty}$ is the freestream velocity, $v_{q}$ is the average velocity of the air permeation through the fabric, $n_{1}, n_{2}$, and $n_{3}$ are the velocity correction coefficients at the inlet, vent, and geometric porosity, respectively, $\alpha$ is the geometric porosity, $r_{1}$ is the radius of the reefing line, $r_{2}$ is the radius of the upper part of the canopy, $r_{d}$ is the radius of the vent, as shown in Figure 1. For the velocity at the geometric porosity, only that at the top of the canopy has the same magnitude of order as the freestream velocity. Thus, only the area of the top of the canopy is contained in the fourth item on the right side of Eq. (13). In general, $v_{q} / v_{\infty}<0.01$, so the second item on the right side of Eq. (13) can be ignored. To maintain the shape of the canopy, the air volume of the inflow must be greater than that of the outflow, that is

$$
\frac{\mathrm{d} V}{\mathrm{~d} t}=n_{1} \pi r_{1}^{2} v_{\infty}-n_{2} \pi r_{d}^{2} v_{\infty}-n_{3} v_{\infty} 2 \pi r_{2}^{2} \alpha \geq 0,
$$

and $r_{2} \geq r_{1}$, so

$$
r_{1} \geq \sqrt{\frac{n_{2}}{\left(n_{1}-2 n_{3} \alpha\right)}} r_{d} .
$$

The inlet radius of the reefed parachute is decided by the reefing ratio:

$$
r_{1}=\beta r_{b},
$$

where $r_{b}$ is the radius of the canopy skirt and $\beta$ is the reefing ratio. Hence, Eq. (15) can be converted to

$$
\beta \geq \sqrt{\frac{n_{2}}{\left(n_{1}-2 n_{3} \alpha\right)}} \frac{r_{d}}{r_{b}}=\beta_{m e},
$$

which is the empirical formula to calculate the minimal effective reefing ratio $\beta_{m e}$. According to Eq. (17), reducing the outlet area, such as by reducing the vent radius and geometric porosity, can enlarge the minimal effective reef-

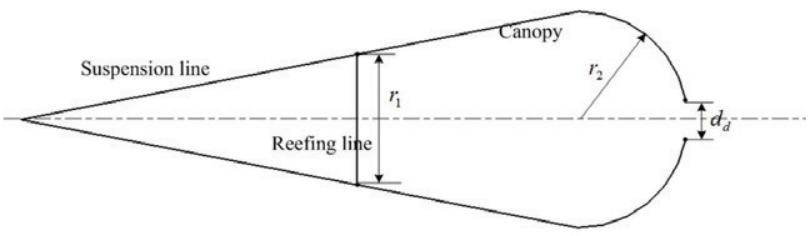

Figure 1. Aerodynamic shape of a reefed parachute ing ratio. In this equation, $n_{1}, n_{2}$, and $n_{3}$ are the empirical parameters, which are determined by the canopy shape and boundary conditions of the flow field. The empirical formula is easy to use in engineering. However, the accuracy of the value is determined by empirical parameters, and these parameters are hard to obtain experimentally. The value of $n_{1}$ equals the average inlet velocity divided by the freestream velocity. The value of $n_{2}$ equals the average vent velocity divided by the freestream velocity. The value of $n_{3}$ equals the average velocity at the canopy top part with geometric porosity divided by the freestream velocity. The minimal effective reefing ratio, $\beta_{m e}$, is obtained by the empirical formula in Eq. (17). The average inlet velocity, average vent velocity, and average velocity at the top part of the canopy with geometric porosity, are obtained by the FSI method.

\section{Geometry and FSI models}

The geometrical parameters of the conical ringslot parachute model are shown in Table 1. Two parachutes with different vent diameters were used in this paper to determine the influence of the outlet area on the effective reefing ratio. The vent diameters of Parachute A and B were $500 \mathrm{~mm}$ and $400 \mathrm{~mm}$, respectively. The numerical mesh of the parachute system is shown in Figure 2. The canopy is petal-shaped from overlooking after direct folding (Ma, Yue, \& Huang, 2005; Cheng, Yu, \& Yin, 2012b). For a reefed parachute, there is a line along the parachute

\begin{tabular}{|c|c|c|c|c|c|c|}
\hline 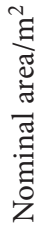 & 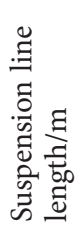 & 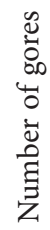 & 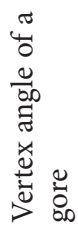 & 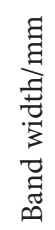 & 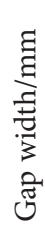 & 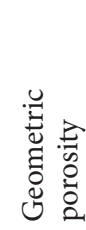 \\
\hline 24 & 7 & 24 & $14^{\circ}$ & 120 & 35 & $18.1 \%$ \\
\hline
\end{tabular}
skirt that constrains the diameter, so the diameter has to be shorter than the diameter in the subsequent stage. In the simulation model, it was folded below the canopy.

Table 1. Geometrical parameters of the conical ringslot parachute model

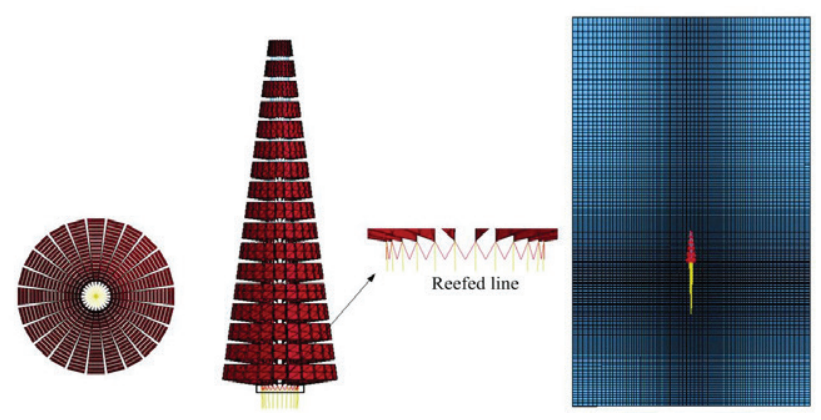

a) Structure mesh

b) Fluid mesh

Figure 2. Parachute system 
Some nodes on the cable were shared with the canopy. The canopy was meshed by about 11,000 triangular elements. The suspension lines, reinforcement belt, and reefing line, were meshed by about 8,000 bar elements. Over 1,890,000 Cartesian elements were used to mesh the flow field. The mesh was refined at the parachute system and wake flow. The computational domain along the axial, normal, and lateral direction of the parachute, was $8 \mathrm{D} 0 \times 4 \mathrm{D} 0 \times 4 \mathrm{D} 0$ (D0 is the canopy nominal diameter, where the wake length is about 4D0). To limit the Courant number to about 0.7 , the time step was set to $6.7 \mathrm{e}-6$. The initial conditions were set to be equal to the freestream values. The nonslip boundary condition was imposed on the coupling surface. The intersection point of the suspension lines was fixed. Fabric porosity was tested through the Ergun equation:

$$
\Delta P=\left(a v_{q}+b v_{q}^{2}\right) e,
$$

where $\Delta P$ is the differential pressure from the canopy fabric test, and $e$ is the thickness of the canopy. Through the porosity test, the viscosity coefficient $a$ and inertia coefficient $b$ of these two fabrics were obtained: $a=7.77 \times 10^{6} \mathrm{~kg} / \mathrm{m}^{3} \cdot \mathrm{s}, b=1.12 \times 10^{7} \mathrm{~kg} / \mathrm{m}^{4}$.

\section{Results and discussion}

In order to validate the FSI method, a comparison was made with experimental data. The experiments were performed in a wind tunnel, with the internal dimensions of $6 \mathrm{~m}$ wide, $6 \mathrm{~m}$ deep, and $8 \mathrm{~m}$ long. Parachutes $\mathrm{A}$ with reefing ratios of $5 \%$ and $25 \%$ were used in the experiment. The reefing line lengths were $268 \mathrm{~mm}$ and $1336 \mathrm{~mm}$. The dynamic pressure was 2, $800 \mathrm{~Pa}$. The opening load and drag area were compared with the experimental results, as depicted in Table 2. The numerical results of these cases were in good agreement with the wind tunnel. The maximum error was $9.3 \%$.
The changes of the canopy shape in the inflation process are shown in Figure 3. The stable shape in the simulation was similar with the wind tunnel results. The shape of the canopy with a reefing ratio of $5 \%$ changed sharply and randomly. The fabric collapsed, and the top of the canopy did not stretch well. The canopy with a reefing ratio of $25 \%$ inflated successfully with its vent and skirt opened. The two states described above are typical examples of an ineffective and effective inflation of a reefed parachute.

The velocity vector and pressure contour of the canopy with a reefing ratio of $5 \%$ and $25 \%$ are shown in Figure 4. When the reefing ratio was $5 \%$, only a small amount of air could enter the canopy, because the inlet was too small. The remaining air with high dynamic pressure stagnated at the inlet and formed an abnormal high-pressure region. The air in the canopy had low dynamic pressure. The internal and external pressure of the canopy had similar values, so the canopy could not form a stable aerodynamic shape easily. The collapsed fabric in turn reduced the inflow of air into the canopy. The canopy shape changed randomly due to the random distribution of the turbulent vortexes. When the reefing ratio was $25 \%$, a small amount of air stagnated at the inlet and formed a high-pressure region. Most of the air entered the canopy and formed two vortexes with opposite directions. This air stagnated at the vent and formed the second high-pressure region. This ensured that the vent was fully opened. In summary, the open degree of the inlet is not affected by the reefing ratio. However, the vent cannot open well when the reefing ratio is too small. Thus, the open degree of the vent is used to judge whether the reefing ratio is effective or not.

With regards to the asymmetry of the canopy, four evenly distributed monitoring points were selected at the inlet of the canopy. In order to analyze the open state and stability of the canopy shape quantitatively, the variation coefficients of the inlet were used:

Table 2. Comparison of the numerical and wind tunnel results

\begin{tabular}{|c|c|c|c|c|c|}
\hline \multirow{2}{*}{ Reefing ratio (\%) } & \multicolumn{2}{|c|}{$F / K N$} & \multicolumn{2}{|c|}{$C A / \mathrm{m}^{2}$} & \multirow{2}{*}{ Error (\%) } \\
\cline { 2 - 6 } & FSI & test & FSI & 0.43 & 9.3 \\
\hline $5 \%$ & 1.330 & 1.204 & 0.47 & 4.79 & 2.5 \\
\hline $25 \%$ & 13.084 & 13.41 & 4.67 & & \\
\hline
\end{tabular}

$5 \%$

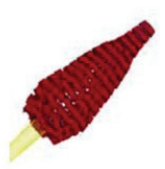

$25 \%$
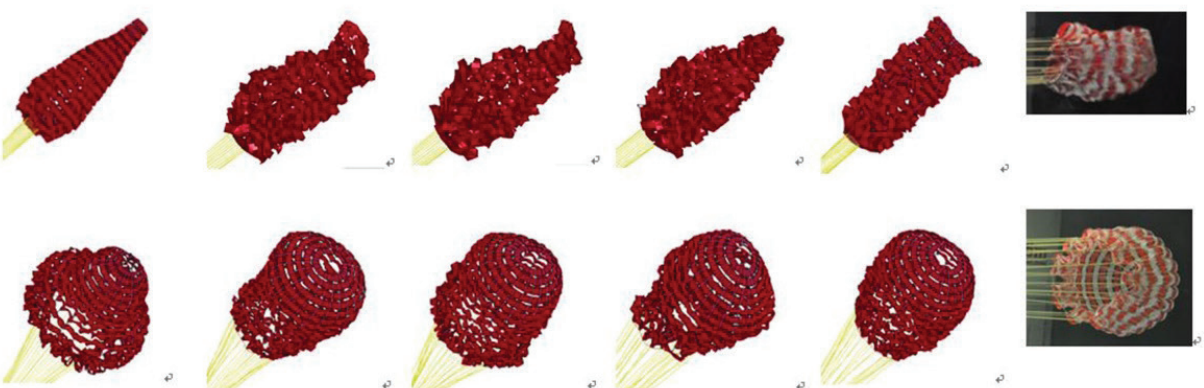

Figure 3. Change of the canopy shape during inflation $(t=0.1,0.3,0.5,0.7,0.9 \mathrm{~s})$ 

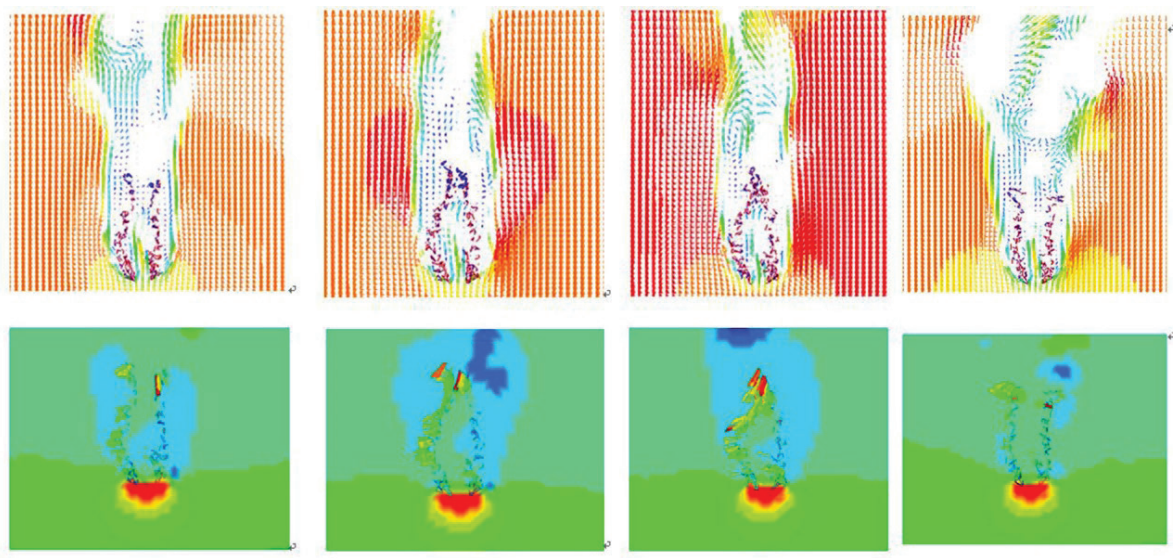

a) Reefing ratio $=5 \%$
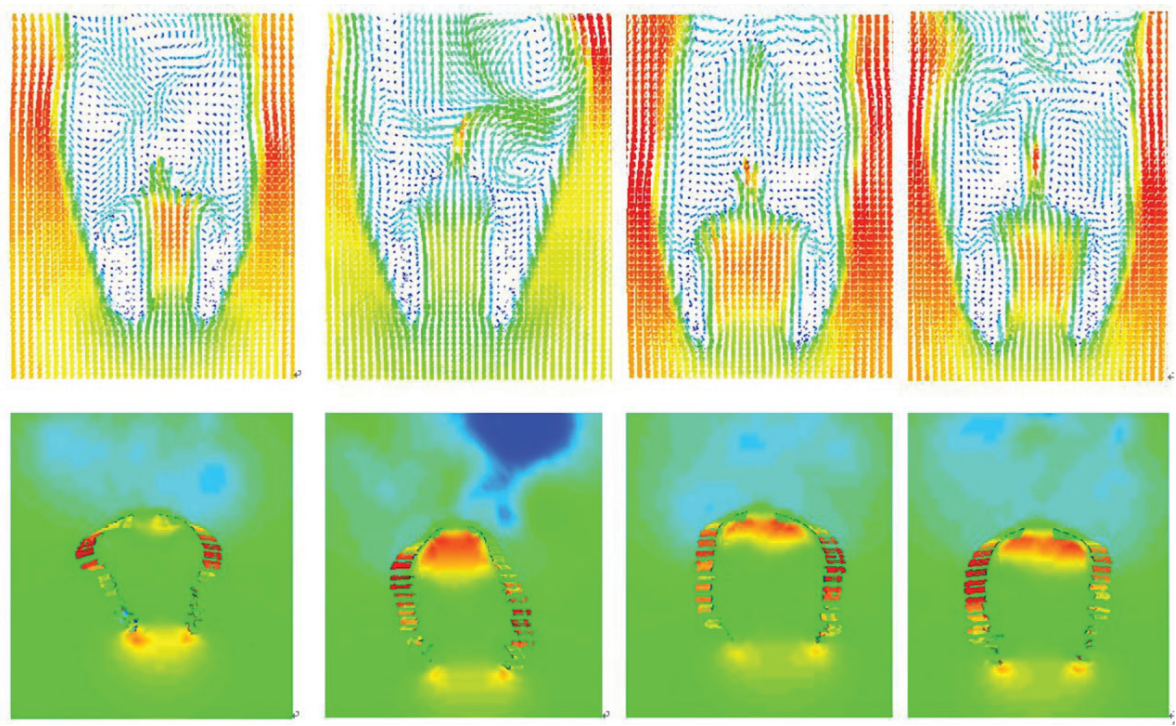

b) Reefing ratio $=25 \%$

Figure 4 . The velocity vector and pressure contour of the canopy at the plane of symmetry $(\mathrm{t}=0.45,0.55,0.65,0.8 \mathrm{~s})$

$$
C_{s}=\frac{\sigma_{s}+\sigma_{t}}{D_{\text {ave }}},
$$

where $D_{\text {ave }}$ is the averaged diameter and

$$
D_{\text {ave }}=\frac{\sum_{1}^{n} D_{e q u}(t)}{n_{i}},
$$

where $n_{i}$ is the number of time samples after inflation, $D_{\text {equ }}(t)$ is the averaged value of the four monitor diameters at the inlet, and

$$
D_{\text {equ }}(t)=\frac{1}{4} \sum_{i=1}^{4} D_{i}(t) .
$$

In Eq. (19), $\sigma_{s}$ and ${ }_{k_{1} \neq k_{2}}$ are the mean squared error of space and time,

$$
\sigma_{t}=\sqrt{\frac{\sum\left(D_{a v e}-D_{e q u, i}\right)^{2}}{n_{i}}},
$$

$$
\sigma_{p}=\sqrt{\frac{\sum \sigma_{p, i}}{n_{i}}},
$$

where $\sigma_{p, i}$ is the mean squared error of space at each time sample, and

$$
\sigma_{p, i}=\sqrt{\frac{\sum\left(D_{i}-D_{e q u, i}\right)^{2}}{4}} .
$$

The numerical results of the variation coefficients at the inlet are shown in Figure 5. The legend in this figure can be interpreted as follows: "1800pa-A" means Parachute A was simulated with a dynamic pressure of $1,800 \mathrm{~Pa}$, and so on. The canopy shape of Parachute A with a dynamic pressure of 2,800 $\mathrm{Pa}$ is also shown in this figure. The plumpness of the canopy was in direct proportion to the reefing ratio. The variation coefficient represents whether the canopy has a stable aerodynamic shape or not, so the 
variation coefficient can be used to judge the effectiveness of the reefing ratio. In Figure 5, the variation coefficient curve was divided into two parts at the turning point between monotonic decreasing and harmonic oscillation. Before the turning point, the stability of the vent improved significantly with the increase of the reefing ratio. The reefing ratios before the turning point were considered to be ineffective. When the parachute was reefed ineffectively, the variation coefficient had a lot of randomness and had

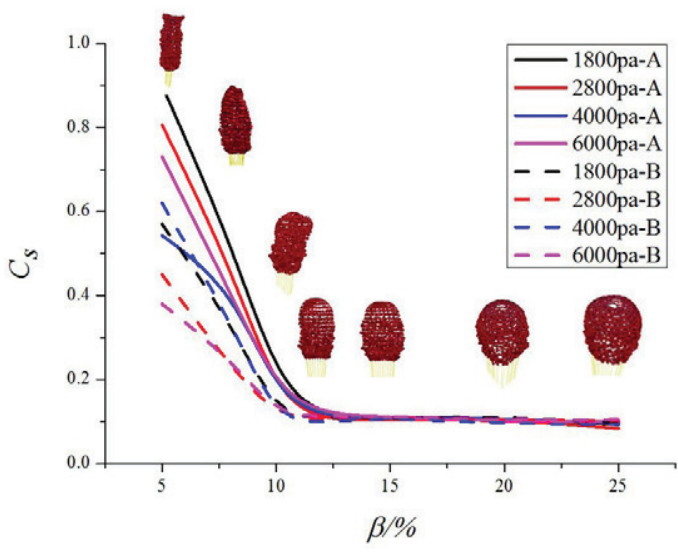

Figure 5. Numerical results of the variation coefficients at the inlet

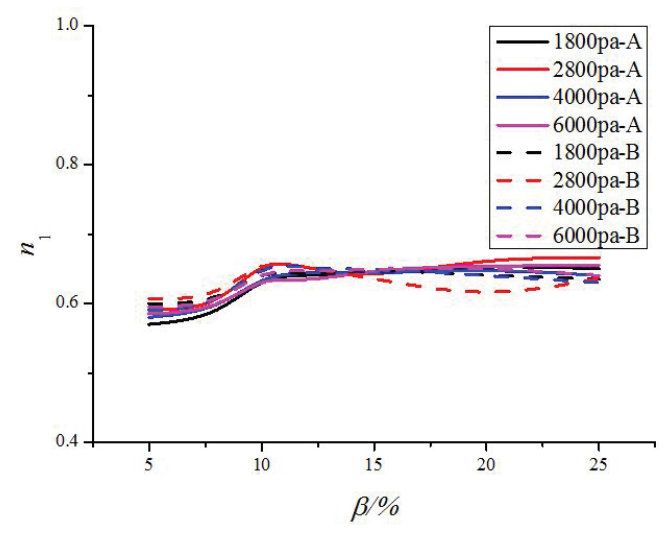

a) $n_{1}$

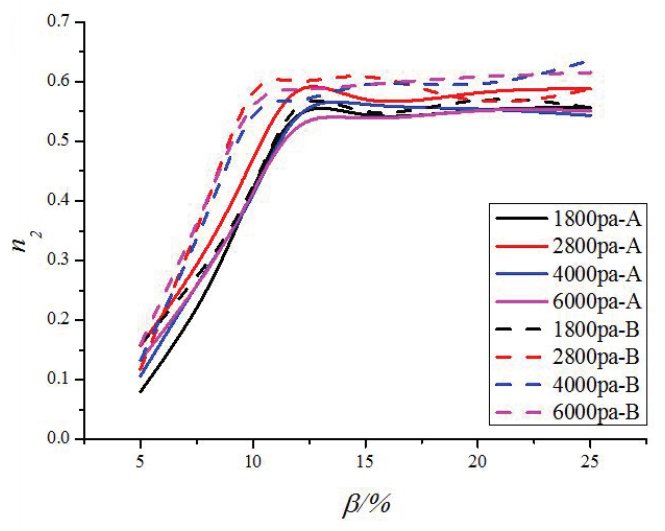

c) $n_{3}$ different values for different dynamic pressures. After the turning point, the difference ratio was less than $15 \%$, so the variation coefficient was slightly affected by the reefing ratio. The reefing ratios after the turning point were considered to be effective. The turning points of Parachute A and $\mathrm{B}$ were $12 \%$ and $10 \%$, respectively. This means that the minimal effective reefing ratios of Parachute $\mathrm{A}$ and $\mathrm{B}$ were $12 \%$ and $10 \%$, respectively. The minimal effective reefing ratio decreased with the decrease of the vent diameter. For the same parachute system, the minimal effective reefing ratio is not affected much by the dynamic pressure.

In order to analyze the accuracy of the empirical formula in Eq. (17), the numerical results of the values in this equation are illustrated in Figure 6. The value of $n_{1}$ is equal to the average inlet velocity divided by the freestream velocity. It is a dimensionless number used to describe the inlet velocity. Based on the analysis, the inlet can open well whether the reefing ratio is effective or not, so $n_{1}$ is affected less by the reefing ratio. The dynamic pressure also has little effect on $n_{1}$. The value of $n_{2}$ is equal to the average vent velocity divided by the freestream velocity. When the reefing ratio is ineffective, $n_{2}$ increases sharply with the reefing ratio, because the flow flux in the canopy is in direct proportion to the reefing ratio. When the reefing ratio is effective, $n_{2}$ is stable. $n_{2}$ increases with the

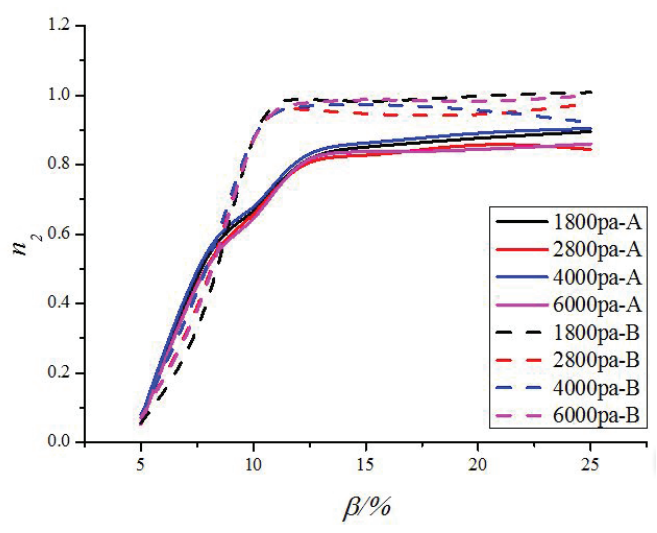

b) $n_{2}$

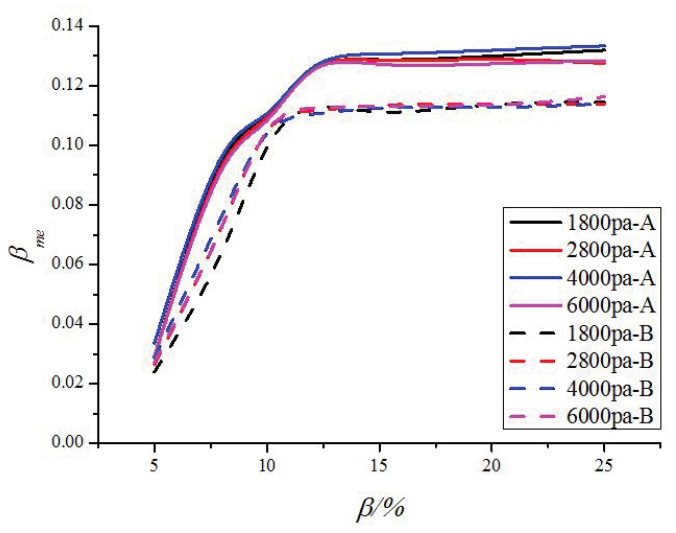

d) $n_{4}$

Figure 6. Numerical results of parameters in the empirical formula (Eq. (17)) 
decrease of the vent diameter. The value of $n_{3}$ is equal to the average velocity at the top part of the canopy with geometric porosity divided by the freestream velocity. Although this part is near the vent, its velocity is lower than the vent's velocity due to the existence of vortexes in the canopy. The law of the development of $n_{3}$ is similar to that of $n_{2}$. The minimal effective reefing ratio, $\beta_{m e}$, is obtained by the empirical formula in Eq. (17). The stable values of $\beta_{m e}$ for Parachute $A$ and $B$ were about $13.1 \%$ and $11.3 \%$, respectively. This indicates that the empirical formula has high accuracy when the values of the empirical parameters are appropriate. $\beta_{m e}$ is larger than that obtained from the numerical simulation due to some simplifying assumptions. The empirical parameters can be obtained by simulating a numerical model of an effectively reefed parachute.

\section{Conclusions}

The main contribution of this paper is to provide a design methodology for the minimum effective reefing ratio based on an empirical formula and the FSI method. The typical flow and structure fields of effective and ineffective reefed parachutes are obtained by the ALE/FSI method. There are two high-pressure regions at the inlet and vent of a parachute system with an effective reefing ratio, while there is only one high-pressure region at the inlet of a parachute system with an ineffective reefing ratio. The open degree of the vent can be used to judge whether the reefing ratio is effective or not. The curve to describe the opening degree of the vent is divided into two parts at the turning point between monotonic decreasing and harmonic oscillation, in response to the increase of the reefing ratio. The turning point is the minimal effective reefing ratio. The minimal effective reefing ratio is affected less by the dynamic pressure; however, it is greatly affected by the geometric porosity. The performance of the empirical formula is discussed by analyzing the numerical results of empirical parameters. The empirical formula has high accuracy when the values of the empirical parameters are appropriate. The empirical parameters can be obtained by simulating a numerical model of an effectively reefed parachute.

In this preliminary study, only one kind of parachute is tested to evaluate whether the proposed methodology works efficiently. Although the results are quite good, tests for other kinds of parachutes remain as a future work. An empirical formula for parachute clusters should be obtained, because they are widely used in multi-stage reefed parachute systems. The empirical parameters rely on the FSI simulation, so the accuracy of the empirical formula relies on the FSI simulation. Other ways to obtain empirical parameters should be studied in the future.

\section{References}

Behr, V., Wolf, D., \& Rutledge, B. (2001). Development of an 80-ftdiameter ribbon drogue parachute for the NASA X-38 vehicle. Paper presented at the 16th Aerodynamic Decelerator Systems Technology Conference and Seminar, Boston, Massachusetts (AIAA 2001-2042). https://doi.org/10.2514/6.2001-2042

Bledsoe, K., Englert, M., Morris, A., \& Olmstead, R. (2009). Overview of the Crew Exploration Vehicle Parachute Assembly System (CPAS) Generation I Main and cluster development test results. Paper presented at the 20th AIAA Aerodynamic Decelerator Systems Technology Conference and Seminar, Seattle, Washington (AIAA 2009-2940).

Cheng, H., Yu, L., Rong, W., \& Jia, H. (2012a). A numerical study of parachute inflation based on a mixed method. Aviation, 16(4), 115-123. https://doi.org/10.3846/16487788.2012.753676

Cheng, H., Yu, L., \& Yin, Z. W. (2012b). A new method of complicated folded fabric modeling. Journal Harbin Institute Technology, 19(2), 43-73.

Gao, S. Y., \& Yu, L. (2014). Influence of reefing ratio on inflation performance of ringsail parachute. Chinese Space Science and Technology, 1(1), 63-70.

Holt, I. T. (1968). Design and development of a heavy duty 76-ft ribbon parachute. Paper presented at the AIAA 2nd Aerodynamic Deceleration Systems Conference (AIAA 68-930). https://doi.org/10.2514/6.1968-930

Jason, D. C. (2009). Computational aerodynamics modeling of the reefed stages of ringsail parachutes. Rice University, Houston.

Jose, G. V., \& Eric, S. R. (2013). Skipped stage modeling and testing of the CPAS Main parachutes. Paper presented at the AIAA Aerodynamic Decelerator Systems (ADS) Conference, Daytona Beach, Florida.

Kenji, T., Tayfun, E. T., \& Cody, B. (2014). FSI modeling of the reefed stages and disreefing of the Orion spacecraft parachutes. Computational Mechanics, 54(5), 1203-1220. https://doi.org/10.1007/s00466-014-1052-y

Ma, C. S., Yue, H., \& Huang, S. L. (2005). A study on airbag folding patterns for improving occupant protection effectiveness. Automotive Engineering, 27(3), 350-362.

Manley, C., \& Butler, J. R. (2009). The design and development of emergency parachute canopies utilizing canopies utilizing the BAT Sombrero Slider. Retrieved from https://www.butlerparachutes.com/pdf/piapresen.pdf

Robert, B. W. (1973). Apollo Experience Report earth landing system (NASA Technical Notes, NASA TN D-7437). Washington.

Runkle, R. E., \& Wolf, D. F. (1995). Space Shuttle Solid Rocket Booster lightweight recovery system. Journal of Spacecraft \& Rockets, 22(6), 668-670. https://doi.org/10.2514/6.1995-1594

Tang, J., \& Qian, L. (2015). Numerical study of the reefing control of parachute inflation shock. Paper presented at the Proceeding of the 2015 IEEE International Conference on Information and Automation in Lijiang, China. https://doi.org/10.1109/ICInfA.2015.7279619

Wang, L. R. (1997). Parachute theory and applications. Beijing, China: Aerospace Press. 